\title{
Elucidating the mechanism by which regulating spleen and lipid method improves the state of hyperlipidaemic rats
}

\section{Guirong Liu (D 645173627@qq.com )}

Shandong University of Traditional Chinese Medicine https://orcid.org/0000-0001-9827-6811

\section{Linlin Pan}

Shandong University of traditional Chinese medicine

\section{Qihui Sun}

Anhui University of Traditional Chinese Medicine

\section{Song Wang}

Shandong University of Traditional Chinese Medicine

\section{Research}

Keywords: the regulating spleen and lipid decoction (RSLD), hyperlipidemia, metabolomics, network pharmacology, UPLC-ESI-QTOF-MS

Posted Date: December 30th, 2019

DOI: https://doi.org/10.21203/rs.2.19568/v1

License: (9) (1) This work is licensed under a Creative Commons Attribution 4.0 International License. Read Full License 


\section{Abstract}

Backgound Hyperlipidemia has been highlighted as global chronic conditions of our time, the regulating spleen and lipid decoction (RSLD) is a traditional Chinese medicine preparation for hyperlipidemia with good therapeutic effect. This study used urinary metabolomics combined with network pharmacology to elucidate the mechanism of RSLD in treating hyperlipidemia.

Methods In this study, the network pharmacology was used to dissect the impacts of RSLD on the hyperlipidemia network. Serum samples from hyperlipidaemic rats were collected to quantify biochemical indices, urinary metabolomics was used to evaluate urine samples from control, model, xuezhikang and RSLD groups to shed light on a metabolic mechanism of action.

Results The component- target-disease network suggested that the low density lipoprotein receptor (LDLR) target protein, which is associated with hyperlipidemia, is regulated by RSLD. A metabolomics analysis suggested that RSLD can significantly regulates taurine, citric acid, leucine, valine and linoleic acid in amino acid metabolism, fatty acid metabolism and tricarboxylic acid cycle, and that the overall metabolic mechanism is closely related to the target protein LDLR.

Conclusions Metabolomics combined with network pharmacology suggested that RSLD can improve hyperlipidemia by enhancing LDLR protein expression to regulate disordered biomarkers and their metabolic pathways. Therefore, we believe that RSLD represents an interesting candidate for further characterization as a treatment for hyperlipidemia.

\section{Background}

Hyperlipidemia is mainly characterized by dyslipidemia, which is defined as an increase in serum levels of triglyceride (TG), total cholesterol (TC), low-density lipoprotein cholesterol (LDL-C) and decrease in level of high-density lipoprotein cholesterol (HDL-C) in the serum [1]. Strikingly, in 2017, hyperlipidemia contributed to half of global mortality [2]. The danger of hyperlipidemia arises out of its causal role in the induction of coronary heart disease, atherosclerosis, pancreatitis, hepatic steatosis and diabetes [3, 4]. Statins have been widely used in treating hyperlipidemia, but are also associated with occasional hepatotoxicity, myalgia, and rhabdomyolysis $[5,6]$. Therefore, further exploration of the pathogenesis of hyperlipidemia and potential additional effective therapeutics are urgently needed.

Traditional Chinese medicine (TCM) theory and related research suggests that the spleen has an important function in metabolising and processing nutritive elements (including lipids) following the injections of food [7]. Further, the importance of spleen in lipid metabolism has been elucidated in a number of preclinical studies [8, 9]. Therefore, if the spleen's core functions are dysregulated, systemic metabolism and excretion of lipids is impacted, leading to hyperlipidemia. As a result, the spleen plays a fundamental role in the occurrence of hyperlipidemia. The regulating spleen and lipid decoction (RSLD) is a TCM preparation that has been used to treat hyperlipidemia for decades, created by professors of this research group. RSLD contains Panax Ginseng C. A. Mey (Renshen), Polygonati Rhizoma (Huangjing), 
Radix Puerariae (Gegeng), Alisma Orientale (Sam.) Juz. (Zexie), Atractylodes Lancea (Thunb.)Dc. (Cangzhu) and Crataegus pinnatifida Bunge (Shanzha), and is thought to improve overall lipid metabolism and adjust the pathological mechanisms of dyslipidemia from multiple factors.

Although the effects of RSLD on hyperlipidemia have been verified in both clinical and animal experiments by members of our research group $[10,11]$, it is not clear which components of the concoction contribute to its therapeutic effect or the specific mechanism for improving hyperlipidemia. Therefore, we used network pharmacology to dissect the impacts of RSLD on the hyperlipidemia network and Ultra-performance liquid chromatography- quadrupole-time-of-flight mass spectrometry (UPLC-QTOF-MS)-based urinary metabolomics to identify potential reliable biomarkers and metabolic pathways for hyperlipidemia. In summary, we combined metabolomics with network pharmacology to explore the mechanism of action of RSLD in treating hyperlipidemia.

\section{Materials And Methods}

\subsection{Network target prediction}

Twenty-two main compounds in RSLD were selected for prediction of biological targets using the Lab of Systems Pharmacology database (http://tcmspw.com/). Then the SwissTargetPrediction database (http://www. swisstargetprediction .ch/) was used to obtain the Uniprot ID of predicted targets. The biological targets associated with hyperlipidemia were chosen from the GAD (https:// geneticassociationdb.nih.gov/) and GeneCards (https://www. genecards.org/) database. Finally, the Cytoscape 3.6.1 software (Bethesda, MD, USA) was used to establish the components-targets-disease network.

\subsection{Animals and feeding}

Male Wistar rats (body mass: $200 \pm 20 \mathrm{~g}$ ) were purchased from Jinan Pengyue Experimental Animal Breeding Co. Ltd., and were housed under specific pathogen-free conditions. RSLD cotains Renshen (6 g), Huangjing (15 g), Gegen (30 g), Zexie (30 g), Cangzhu (15 g), and Shanzha (15 g). First, these drugs (Jianlian Chinese Medicine Co. Ltd., China) were pulverized to a superfine powder, then dissolved in 111 $\mathrm{ml}$ water (10x total drug weight) and heated to reflux for 40 minutes. Next, another $111 \mathrm{ml}$ of water was added for another 40 minutes of reflux after filtering, and the solution was concentrated using a rotary evaporator at $50^{\circ} \mathrm{C}$. Finally, RSLD was prepared to a final total concentration of $3 \mathrm{~g}$ drug product $/ \mathrm{ml}$ and stored at $4^{\circ} \mathrm{C}$.

\subsection{Reagents and equipment}

Liquid chromatography was performed using the 2777C UPLC SYSTEM (Waters, UK) with a diode array detector. In this work, we used a mass spectrometer (Xevo G2-XS QTOF, Waters, UK), 3-18K cryogenic high-speed centrifuge (Sigma Company, Germany), automatic biochemical analyzer (Vital Scientific, Netherlands), high-speed centrifuge (Shanghai Medical Analyzer, China), reagents for detecting blood 
lipid (Nanjing Jiancheng Biotech Inc., China), Western blotting (Wuhan Servicebio Biotechnology Co., Ltd, China), methanol, formic acid, acetonitrile, Milli-Q ultrapure water system, deuterated reagent, High fat diet (HFD, $10 \%$ lard, $10 \%$ egg yolk powder, $2 \%$ cholesterol, $0.2 \%$ bile salt and $0.2 \% 6$-methyl-2-thiouracil. Keao Co., Ltd , Beijing, China), xuezhikang (XZK, Beida Weixin Co., Ltd, China, Chinese medicine number: Z10950029).

\subsection{Establishment of the hyperlipidemia model}

Hyperlipidemia rat model was established based on the method of Ji et al 2018 [12]. Briefly, rats were housed in separate cages with a normal diet and free access to water at the temperature of $23^{\circ} \mathrm{C}, 50 \%$ relative humidity, and a $12 \mathrm{~h}$ light-dark cycle. After one week, the rats were randomly divided into control group $(n=8)$ fed with the general diet, and hyperlipidemia model group fed with HFD every day. After 5 weeks, the rats were fasted for $12 \mathrm{~h}$, and then blood samples were taken from the tail for measurement of blood lipid levels in order to verify a successful model of hyperlipidemia. Finally, the animals were randomly divided into model group $(n=8)$, XZK group $(n=8)$ and RSLD group $(n=8)$.

\subsection{Dosing measurement design}

The control group was given saline $(0.5 \mathrm{ml} / 100 \mathrm{~g})$, model, XZK and RSLD groups were given HFD (0.5 $\mathrm{ml} / 100 \mathrm{~g})$ every morning. In the afternoon, the control group was given saline $(0.5 \mathrm{ml} / 100 \mathrm{~g}$, according to their body weight), the XZK group was given XZK $(0.5 \mathrm{ml} / 100 \mathrm{~g})$ and the rats in the RSLD group was administered RSLD $(0.5 \mathrm{ml} / 100 \mathrm{~g}$, according to the transforming formula= (daily dose of RSLD in human (g))/human weight $(\mathrm{kg})) \times 9.1$, the usual human weight is $60 \mathrm{~kg}$ in China and $40 \mathrm{~g} / \mathrm{d}$ is the daily dose in humans, so the rat dose is $6 \mathrm{~g} / \mathrm{d}$ ) from the $6^{\text {th }}$ week to $9^{\text {th }}$ week.

\subsection{Sample preparation}

After 4 weeks treatment, each rat was placed alone in a metabolic cage to collect urine samples for 24 hours. Next, $40 \mu \mathrm{l}$ of urine was added in a microcentrifuge tube, followed by the addition of $120 \mu \mathrm{l}$ of cold methanol, and centrifugation at 3,500 rmp for $10 \mathrm{~min}$. Next, $25 \mu \mathrm{l}$ of supernatant was placed into a new eppendorf tube and diluted with $225 \mu \mathrm{l}$ of $50 \%$ methanol. Finally, $25 \mu \mathrm{l}$ of each sample was mixed into a quality control (QC) sample for liquid chromatography and mass spectrometry analysis. Blood was collected from the carotid artery, and the serum was separated by centrifugation at 3,500rpm for $10 \mathrm{~min}$. Serum was stored at $-80^{\circ} \mathrm{C}$ for further biochemistry examinations.

\subsection{Western blotting}

First, the hepatic tissue was extracted and the total protein was obtained by using RIPA buffer containing PMSF, and then centrifuging at $12000 \mathrm{rpm}$ for $10 \mathrm{~min}$. Second, the nuclear protein was extracted and the concentration was determined by the BCA protein assay. Third, the total protein was subjected to SDSPAGE gel electrophoresis and electroblotted onto a PVDF membrane, and the membrane was blocked in $5 \% \mathrm{BSA}$ for $1 \mathrm{~h}$ and then incubated with the following primary antibody overnight at $4{ }^{\circ} \mathrm{C}$. Antibodies: 
ACTIN (mouse monoclonal antibody 1:1000), low density lipoprotein receptor (LDLR, rabbit monoclonal antibody 1:1000), and then incubated the membrane with 1:3000 secondary antibody and visualized the conjugate using the ECL system. Finally, the protein expression was analyzed using the odyssey imaging system.

\subsection{Chromatography and mass spectrometry conditions}

Firstly, UPLC system was used to perform chromatographic separations. An ACQUITY UPLC HSS T3 column $(100 \mathrm{~mm} \star 2.1 \mathrm{~mm}, 1.8 \mu \mathrm{m})$ was used for reverse phase separation, and the column oven was maintained at $45^{\circ} \mathrm{C}$. The mobile phase using solvent $A$ (water $+0.1 \%$ formic acid) and solvent $B$ (acetonitrile+0.1\% formic acid), the flow rate was set at $0.4 \mathrm{ml} / \mathrm{min}$. Gradient elution conditions were set as follows:0-1 min, $99 \%$ phase $A ; 1-3$ min, $1 \%$ to $15 \%$ phase $B ; 3-6$ min, $15 \%-50 \%$ phase $B ; 6-9$ min, $50 \%$ to $95 \%$ phase $A, 9-10 \mathrm{~min}, 95 \%$ phase $B ; 10-12 \mathrm{~min}, 99 \%$ phase $A$. The injection volume for per sample was $10 \mu \mathrm{l}$.

The high resolution tandem mass spectrometer was used to detect the metabolites eluted from the column. Q-TOF was divided into two modes: positive ion and negative ion. The sample cone and capillary voltage were set at $2.5 \mathrm{kv}$ and $60 \mathrm{v}$ in the positive (POS) ion mode, and $2.0 \mathrm{kv}$ and $40 \mathrm{v}$ in the negative (NEG) ion mode, respectively. MS data was obtained in centroid mode from 50 to 1200 da with 0.2 $\mathrm{s} / \mathrm{scan}$. The precursors were fragmented using $20-40 \mathrm{eV}$ in MS/MS detection with $0.2 \mathrm{~s} / \mathrm{scan}$. In order to evaluate the stability of UPLC-Q-TOF-MS during the acquisition process, one QC sample was taken every 8 samples.

\subsection{Statistical analysis}

The total ion current (TIC) data was collected by UPLC-Q-TOF-MS and the data matrix of mass detection, retention time and peak intensity was processed by peak detection, matching, alignment and control processing. The score plot was obtained by partial least squares discriminant analysis in SIMCA-P12.0. The significant metabolites were selected from the resulting data set by discriminant analysis and multivariate statistical methods. Variable importance projection (VIP) values $>1.0$ and $P$ value $<0.05$ were considered to be potential biomarkers. To check exact molecular weight, two online databases were queried-METLIN (http://www.metlin. scipps.edu) and HMDB (http://www.hmdb.ca) to explain the mass spectra and identify the structure of the compounds.

\section{Results}

\subsection{The network pharmacology analysis}

We set the criterias oral bioavailability $(O B) \geq 30 \%$ and drug-likeness (DL) $\geq 0.18$ to screen the active ingredients in RSLD. Finally, the component-target-disease network indicated that 43 target proteins were closely associated with 22 active components in RSLD ( As shown in Figure 1 and Table S1). In addition, the LDLR target protein was closely related to RSLD in improving hyperlipidemia. 
Figure 1 The component-target-disease network formed after RSLD intervention

\subsection{Body weight and blood lipid level}

We found that, compared with the model group, the body weight of rats in the XZK and RSLD group decreased significantly after treatment (Figure $2, \mathrm{P}<0.01$ ). As shown in Figure 3 , after treatment, the HDL$C$ level was significantly increased in the XZK and RSLD groups $(P<0.01)$, and TG, TC and LDL-C levels in $X Z K$ and RSLD groups were obviously lower than that of model group $(P<0.01)$.

\section{Figure 2 Body weight in each group $\square \pm x \square$}

Note: Compared with the model group at the same time period, ${ }^{*} \mathrm{P}<0.01$.

\section{Figure 3 Blood lipid levels after treatment! $x \pm s \square$}

Note: Compared with the model group at the same time period, ${ }^{*} \mathrm{P}<0.01$.

\subsection{Western blotting}

LDLR plays an important role in improveing hyperlipidemia [13], so the protein expression of LDLR was analyzed in this study. As shown in the result (Figure 4), the protein expression of LDLR in the model group was obviously decreased compared with the control group. While in XZK and RSLD group, the LDLR protein expression was obviously increased compared with the model group.

\section{Figure 4 LDLR protein expression after treatment}

\subsection{Metabolomic analysis}

The overlaid chromatograms in the POS and NEG ion modes demonstrated good reproducibility (Figure 5). The orthogonal partial least-squares discriminant analysis (OPLS-DA) score plot suggested that the inter-groups were well separated and the sample modelling was acceptable (Figure 6). In addition, RSLD group was closer to control than XZK and model groups, which indicated that RSLD has a more powerful therapeutic effect than XZK or saline control. The cluster heat plot showed the different metabolites and their relative increase or decrease of values among different groups, and after treating with RSLD, the levels of the metabolites were close to the control group (Figure 7).

The multivariate data analysis was performed in this study. We screened the metabolites with VIP value > 1 and $\mathrm{P}<0.05$ on the resulting metabolite profile, and then searched these metabolites in the kyoto encyclopedia of genes and genomes (KEGG) database (http: //www.kegg.com). Finally, twenty-six potential metabolites were identified (Table 1). We found that after treatment with RSLD, twenty-two potential biomarkers were upregulated, and four potential biomarkers were downregulated in the urine. The potential metabolic pathways disturbed in hyperglycaemic rats were presented in Figure 8. As shown in Figure 9, the following pathways had strong impacts: linoleic acid metabolism; taurine and hypotaurine 
metabolism; valine, leucine and isoleucine biosynthesis; glyoxylate and dicarboxylate metabolism and tricarboxylic acid (TCA) cycle.

Figure 5 Base peak intensity chromatogram

Figure 6 The OPLS-DA score plot after treatment

Figure 7 The cluster heat plot after treatment

Note: $\mathrm{C}$ refers to control group, $\mathrm{M}$ refers to model group, $\mathrm{R}$ refers to RSLD group, $\mathrm{X}$ refers to XZK group.

Figure 8 Potential metabolic pathways changed in hyperglycemic rats after treatment

Figure 9 The pathways impacts regulated by RSLD

Figure 10 Comparison of the relative intensity of potential biomarkers regulated by LDLR

Note: The blue frame represents the metabolic pathway, and the red frame represents potential biomarkers. Compared with the model group, ' $\uparrow$ 'refers to an upward trend and ' $\downarrow$ 'refers to an downward trend in RSLD group.

Table 1 Biomarkers in the urine of hyperlipidaemic rats

Note: Compared with the model group, ' $\uparrow$ ' refers to an upward trend and ' $\downarrow$ ' refers to a downward trend in the RSLD group.

\section{Discussion}

XZK (Chinese National Food and Drug Administration Standard YBZ01592004), whose main component is red yeast, is an effective antilipidemic drug developed by Chinese biopharmaceutical companies [14, 15] Thus, we chose XZK to compare the lipid-lowering effect of RSLD. We found that the body weight and blood lipid level improved significantly and some metabolites related to lipid metabolism showed the same trend after treatment with XZK and RSLD. Therefore, like XZK, RSLD appears to have a beneficial effect in regulating blood lipid levels.

\subsection{The correlation between metabolomic results and hyperlipidemia}

Abnormal lipid metabolism is the most obvious manifestation of hyperlipidemia. Linoleic acid is a polyunsaturated fatty acid and precursor of prostaglandins, which is helpful in lowering blood lipid [16]. In addition, linoleic acid has been identified via metabolomic profiling as being differentially regulated in hyperlipidemia progression [17]. In this study, compared with the model group, the level of linoleic acid in RSLD group increased obviously, and the impact on the linoleic acid metabolic pathway was significant (Figure 9), suggesting that RSLD may improve hyperlipidemia via a mechanism involving a change in linoleic acid metabolism. 
Leucine and valine are essential amino that act as signalling molecules to control energy homeostasis involving lipid metabolism [18, 19]. Additionally, leucine can regulate fat metabolism, reduce fat synthesis, promote fat decomposition and increase energy consumption in mammals [20,21]. It has been shown that supplementation with leucine can inhibit the action and synthesis of fatty acids, assisting in clinical weight loss $[22,23]$. In this work, compared with the model group, RSLD group led to increased concentrations of leucine and valine, indicating that RSLD may affect amino acid metabolism and regulate fat metabolism.

TCA cycle is responsible for acetyl coenzyme A (CoA) oxidation, releasing energy from fat, protein and sugar [24]. Citric acid is an important intermediate product in TCA cycle linking oxidative metabolism of carbohydrate, protein and fat [25]. Taurine in the liver combines with bile acids to form taurocholic acid, which can increase the solubility of lipids that play an important part in the absorption of lipids in the digestive tract [26,27], and a study found that taurine supplementation led to a reduction of TG, TC and LDL-C in alloxan-induced rats [28]. Uric acid is an important antioxidant involved in the body's acid-base balance in physiology and is closely related to the occurrence of hyperglycaemia [29, 30]. In this work, we also found that the levels of citric acid, taurine and uric acid in hyperlipidemic rats were changed obviously after treatment with RSLD, providing a beneficial effect contributing to the regulation of dyslipidemia.

\subsection{The correlation between network pharmacology and metabolomics}

Prior work has shown that deficiency in LDLR proteins induces hyperlipidemia [13], and the LDLR (-/-) rats model have been widely used to mimic cardiovascular and cerebrovascular diseases [31, 32]. The western blotting suggested that treatment with RSLD appears to enhance LDLR protein expression in the liver. The network pharmacology study showed that the LDLR target protein is associated with RSLD. Previous metabolomic studies have shown that LDLR protein deficiency causes a significant metabolic perturbation in TCA cycle, amino acid metabolism and fatty acid metabolism, and influences levels of taurine, linoleic acid, citric acid, leucine, and valine [33-35]. As shown in Figure 10, we observed similar changes in concentration of taurine, linoleic acid, citric acid, leucine, and valine in these related metabolic pathways. Indeed, we believe that RSLD enhances protein expression of LDLR to restore function of amino acid metabolism, fatty acid metabolism and TCA cycle as a means of attenuating hyperlipidemia.

\section{Conclusion}

RSLD has a good regulation effect on lipid metabolism disorders throug regulating the disordered biomarkers and their metabolic pathways to a normal state. It can improve the environment of lipid metabolism in the whole body, and regulate the pathological mechanism of hyperlipidaemia from multiple links and multiple targets. Therefore, this prescription is effective in treating hyperlipidaemia and has important reference and popularization significance for the clinical treatment of hyperlipidaemia.

\section{Abbreviations}


HDL-C, high-density lipoprotein cholesterol; HFD, high fat diet; KEGG, kyoto encyclopedia of genes and genomes; LDL-C, low-density lipoprotein cholesterol; LDLR, low density lipoprotein receptor; NEG, negative; OPLS-DA, orthogonal partial least-squares discriminant analysis; POS, positive; QC, quality control; RSLD, the regulating spleen and lipid decoction; TC, total cholesterol; TCA, tricarboxylic acid; TCM, traditional Chinese medicine; TG, triglyceride; TIC, total ion current; UPLC-Q-TOF-MS, ultra-performance liquid chromatography- quadrupole-time-of-flight mass spectrometry; VIP, variable importance projection; XZK, xuezhikang.

\section{Declarations}

\section{Ethics approval}

This study was approved by the Animal Ethics Committee of Shandong University of Traditional Chinese Medicine.

\section{Data availability}

The datasets analyzed during the current study are available from the corresponding author on reasonable request.

\section{Conflicts of interest}

The authors declare that have no competing interests.

\section{Consent for publication}

Not applicable.

\section{Authors' contributions}

LP and GL designed the study, LP and SW conducted the study, LP and QS collected and analyzed the data, LP and GL wrote the manuscript. All authors had read and approved the final manuscript.

\section{Funding}

Supported by the State Administration of Traditional Chinese Medicine, Master of Traditional Chinese Medicine Zhang Zhiyuan Studio Construction Project (National Chinese Medicine Office Letter [2018] 119); National Traditional Chinese Medicine Administration Key Discipline Construction Project of Traditional Chinese Medicine (National Chinese Medicine Office Letter [2012] No. 32)

\section{References}

1. Fazio S, Linton MF: The role of fibrates in managing hyperlipidemia: mechanisms of action and clinical efficacy. Curr Atheroscler Rep 2004, 6(2):148-157. 
2. Chen YL, Xiao CH, Hu ZX, Liu XS, Liu Z, Zhang WN, Zhao XJ: Dynamic lipid profile of hyperlipidemia mice. Journal of chromatography $B$, Analytical technologies in the biomedical and life sciences 2017, 1055-1056:165-171.

3. Meex RCR, Watt MJ: Hepatokines: linking nonalcoholic fatty liver disease and insulin resistance. Nature reviews Endocrinology 2017, 13(9):509-520.

4. Mitchell S, Roso S, Samuel M, Pladevall-Vila M: Unmet need in the hyperlipidaemia population with high risk of cardiovascular disease: a targeted literature review of observational studies. $B M C$ cardiovascular disorders 2016, 16:74.

5. Wang Y, Liu D, Li Y, Guo L, Cui Y, Zhang X, Li E: Metabolomic analysis of serum from obese adults with hyperlipemia by UHPLC-Q-TOF MS/MS. Biomedical chromatography : BMC 2016, 30(1):48-54.

6. Taylor BL, Woodfall GE, Sheedy KE, O'Riley ML, Rainbow KA, Bramwell EL, Kellow NJ: Effect of Probiotics on Metabolic Outcomes in Pregnant Women with Gestational Diabetes: A Systematic Review and Meta-Analysis of Randomized Controlled Trials. Nutrients 2017, 9(5).

7. Fatouros $M$, Bourantas $K$, Bairaktari E, Elisaf $M$, Tsolas $O$, Cassioumis $D$ : Role of the spleen in lipid metabolism. The British journal of surgery 1995, 82(12):1675-1677.

8. Simoes FC, Marques RG, Diestel CF, Caetano CE, Dinis AP, Horst NL, Nogueira Neto JF, Portela MC: Lipidic profile among rats submitted to total splenectomy isolated or combined with splenic autotransplant. Acta cirurgica brasileira 2007, 22 Suppl 1:46-51.

9. Ai XM, Ho LC, Han LL, Lu JJ, Yue X, Yang NY: The role of splenectomy in lipid metabolism and atherosclerosis (AS). Lipids in health and disease 2018, 17(1):186.

10. GR L: Study on the treatment of simple dyslipidemia with Lipi Tiaozhi Capsule. Journal of Shandong University of Traditional Chinese Medicine 2002, 26:179-181.

11. GR L: Study on LiPi TiaoZhi Capsule in treating dyslipidemia simplex. Shandong University of Traditional Chinese Medicine (Doctoral Disserta)

12. Ji H, Liu Y, He F, An R, Du Z: LC-MS based urinary metabolomics study of the intervention effect of aloeemodin on hyperlipidemia rats. Journal of pharmaceutical and biomedical analysis 2018, 156:104-115.

13. Zhao Y, Yang Y, Xing R, Cui X, Xiao Y, Xie L, You P, Wang T, Zeng L, Peng W et al: Hyperlipidemia induces typical atherosclerosis development in Ldlr and Apoe deficient rats. Atherosclerosis 2018, 271:26-35.

14. Moriarty PM, Roth EM, Karns A, Ye P, Zhao SP, Liao Y, Capuzzi DM, Bays HE, Zhang F, Liu S et al: Effects of Xuezhikang in patients with dyslipidemia: a multicenter, randomized, placebo-controlled study. Journal of clinical lipidology 2014, 8(6):568-575.

15. Fan XF, Deng YQ, Ye L, Li YD, Chen J, Lu WW, Li JP: Effect of Xuezhikang Capsule on serum tumor necrosis factor-alpha and interleukin-6 in patients with nonalcoholic fatty liver disease and hyperlipidemia. Chinese journal of integrative medicine 2010, 16(2):119-123.

16. Russo GL: Dietary n-6 and n-3 polyunsaturated fatty acids: from biochemistry to clinical implications in cardiovascular prevention. Biochemical pharmacology 2009, 77(6):937-946. 
17. Xu QY, Liu YH, Zhang Q, Ma B, Yang ZD, Liu L, Yao D, Cui GB, Sun JJ, Wu ZM: Metabolomic analysis of simvastatin and fenofibrate intervention in high-lipid diet-induced hyperlipidemia rats. Acta pharmacologica Sinica 2014, 35(10):1265-1273.

18. Yang P, Hu W, Fu Z, Sun L, Zhou Y, Gong Y, Yang T, Zhou H: The positive association of branchedchain amino acids and metabolic dyslipidemia in Chinese Han population. Lipids in health and disease 2016, 15:120.

19. Riera-Borrull M R-GE, Hernández-Aguilera A, Luciano F, Ras R3, Cuyàs E, Camps J, Segura-Carretero A, Menendez JA, Joven J, Fernández-Arroyo S: Journal of the American Society for Mass Spectrometry.

20. Freudenberg A, Petzke KJ, Klaus S: Dietary L-leucine and L-alanine supplementation have similar acute effects in the prevention of high-fat diet-induced obesity. Amino acids 2013, 44(2):519-528.

21. Chen Q, Reimer RA: Dairy protein and leucine alter GLP-1 release and mRNA of genes involved in intestinal lipid metabolism in vitro. Nutrition (Burbank, Los Angeles County, Calif) 2009, 25(3):340349.

22. Zemel MB, Bruckbauer A: Effects of a leucine and pyridoxine-containing nutraceutical on fat oxidation, and oxidative and inflammatory stress in overweight and obese subjects. Nutrients 2012, 4(6):529-541.

23. Lopez N, Sanchez J, Pico C, Palou A, Serra F: Dietary l-leucine supplementation of lactating rats results in a tendency to increase lean/fat ratio associated to lower orexigenic neuropeptide expression in hypothalamus. Peptides 2010, 31(7):1361-1367.

24. Chen M LR, Weng Y: Discovery and enlightenment of the tricarboxylic acid cycle. Medicine and philosophy 2012, 33:71-73.

25. Burdock G: Fenaroli's Handbook of Flavor Ingredients, Fifth Edition. Fenarolis Handbook of Flavor Ingredients. 2010; 311. 2010:311.

26. Warskulat U, Borsch E, Reinehr R, Heller-Stilb B, Monnighoff I, Buchczyk D, Donner M, Flogel U, Kappert G, Soboll S et al: Chronic liver disease is triggered by taurine transporter knockout in the mouse. FASEB journal : official publication of the Federation of American Societies for Experimental Biology 2006, 20(3):574-576.

27. Rull A, Vinaixa M, Angel Rodriguez M, Beltran R, Brezmes J, Canellas N, Correig X, Joven J: Metabolic phenotyping of genetically modified mice: An NMR metabonomic approach. Biochimie 2009, 91(8):1053-1057.

28. Das J, Vasan V, Sil PC: Taurine exerts hypoglycemic effect in alloxan-induced diabetic rats, improves insulin-mediated glucose transport signaling pathway in heart and ameliorates cardiac oxidative stress and apoptosis. Toxicology and applied pharmacology 2012, 258(2):296-308.

29. Horwath-Winter J, Kirchengast S, Meinitzer A, Wachswender C, Faschinger C, Schmut O:

Determination of uric acid concentrations in human tear fluid, aqueous humour and serum. Acta ophthalmologica 2009, 87(2):188-192.

30. Conen D, Wietlisbach V, Bovet P, Shamlaye C, Riesen W, Paccaud F, Burnier M: Prevalence of hyperuricemia and relation of serum uric acid with cardiovascular risk factors in a developing 
country. BMC public health 2004, 4:9.

31. Ishibashi S, Goldstein JL, Brown MS, Herz J, Burns DK: Massive xanthomatosis and atherosclerosis in cholesterol-fed low density lipoprotein receptor-negative mice. The Journal of clinical investigation 1994, 93(5):1885-1893.

32. Ma Y, Wang W, Zhang J, Lu Y, Wu W, Yan H, Wang Y: Hyperlipidemia and atherosclerotic lesion development in Ldlr-deficient mice on a long-term high-fat diet. PloS one 2012, 7(4):e35835.

33. Cheng KK, Benson GM, Grimsditch DC, Reid DG, Connor SC, Griffin JL: Metabolomic study of the LDL receptor null mouse fed a high-fat diet reveals profound perturbations in choline metabolism that are shared with ApoE null mice. Physiological genomics 2010, 41(3):224-231.

34. Vinaixa M, Rodriguez MA, Rull A, Beltran R, Blade C, Brezmes J, Canellas N, Joven J, Correig X:

Metabolomic assessment of the effect of dietary cholesterol in the progressive development of fatty liver disease. Journal of proteome research 2010, 9(5):2527-2538.

35. Li D, Zhang L, Dong F, Liu Y, Li N, Li H, Lei H, Hao F, Wang Y, Zhu Y et al: Metabonomic Changes Associated with Atherosclerosis Progression for LDLR(-/-) Mice. Journal of proteome research 2015, 14(5):2237-2254.

\section{Table}

Table 1. Biomarkers in the urine of hyperlipidaemic rats 


\begin{tabular}{|c|c|c|c|c|c|c|c|}
\hline No & $\begin{array}{c}\text { Identification } \\
\text { result }\end{array}$ & $\begin{array}{l}\text { Molecular } \\
\text { formula }\end{array}$ & $\begin{array}{c}\text { Retention } \\
\text { Time }\end{array}$ & $\begin{array}{l}\text { Measured } \\
\text { Mass }\end{array}$ & $\begin{array}{c}\text { Ionization } \\
\text { mode }\end{array}$ & $\begin{array}{c}\text { VIP } \\
\text { scores }\end{array}$ & Trend \\
\hline 1 & Threonine & C4H9NO3 & 1.15 & 119.0053 & $\mathrm{M}+\mathrm{H}$ & 1.75 & $\uparrow$ \\
\hline 2 & Proline & C5H9NO2 & 3.91 & 116.0607 & $\mathrm{M}+\mathrm{H}$ & 4.90 & $\uparrow$ \\
\hline 3 & Valine & C5H11NO2 & 3.84 & 118.0763 & $\mathrm{M}+\mathrm{H}$ & 1.45 & $\uparrow$ \\
\hline \multirow[t]{2}{*}{4} & Hippuric acid & C9H9NO3 & 3.58 & 180.0502 & $\mathrm{M}+\mathrm{H}$ & 1.91 & $\downarrow$ \\
\hline & Aspartic acid & $\mathrm{C} 4 \mathrm{H} 7 \mathrm{NO} 4$ & 4.18 & 134.0450 & $\mathrm{M}+\mathrm{H}$ & 1.24 & $\uparrow$ \\
\hline \multicolumn{8}{|l|}{5} \\
\hline 6 & Tryptophan & $\mathrm{C} 11 \mathrm{H} 12 \mathrm{~N} 2 \mathrm{O} 2$ & 3.65 & 205.0970 & $\mathrm{M}+\mathrm{H}$ & 2.97 & $\uparrow$ \\
\hline 7 & L-Cysteine & $\mathrm{C} 11 \mathrm{H} 14 \mathrm{~N} 2$ & 3.72 & 122.0261 & $\mathrm{M}+\mathrm{H}$ & 2.42 & $\uparrow$ \\
\hline 8 & Glutaurine & C7H14N2O6S & 9.40 & 255.0657 & $\mathrm{M}+\mathrm{H}$ & 2.39 & $\uparrow$ \\
\hline 9 & Phenylpropanoate & $\mathrm{C} 9 \mathrm{H} 10 \mathrm{O} 2$ & 3.23 & 151.0756 & $\mathrm{M}+\mathrm{H}$ & 2.13 & $\uparrow$ \\
\hline 10 & $3,4-$ & $\mathrm{C} 7 \mathrm{H} 6 \mathrm{O} 4$ & 8.92 & 155.0343 & $\mathrm{M}+\mathrm{H}$ & 1.92 & $\uparrow$ \\
\hline & Dihydroxybenzoate & & & & & & \\
\hline 11 & D-Aspartate & $\mathrm{C} 4 \mathrm{H} 7 \mathrm{NO} 4$ & 4.18 & 134.0449 & $\mathrm{M}+\mathrm{H}$ & 1.24 & $\uparrow$ \\
\hline 12 & N-Methyltryptamine & $\mathrm{C} 11 \mathrm{H} 14 \mathrm{~N} 2$ & 4.15 & 175.1226 & $\mathrm{M}+\mathrm{H}$ & 3.27 & $\uparrow$ \\
\hline 13 & Citrulline & C6H13N3O3 & 0.83 & 174.0873 & $\mathrm{M}-\mathrm{H}$ & 1.75 & $\uparrow$ \\
\hline 14 & Oxoglutaric acid & C5H6O5 & 0.56 & 145.0134 & $\mathrm{M}-\mathrm{H}$ & 1.59 & $\uparrow$ \\
\hline 15 & Salicylic acid & C7H6O3 & 3.84 & 137.0233 & $\mathrm{M}-\mathrm{H}$ & 1.45 & $\uparrow$ \\
\hline 16 & $\begin{array}{l}\text { alpha- } \\
\text { Isopropylmalate }\end{array}$ & C7H12O5 & 3.27 & 175.0600 & $\mathrm{M}-\mathrm{H}$ & 1.57 & $\uparrow$ \\
\hline 17 & 3-Dehydroquinate & C7H10O6 & 3.05 & 189.0393 & M-H & 1.51 & $\uparrow$ \\
\hline 18 & Noradrenaline & C8H11NO3 & 3.63 & 168.0657 & $\mathrm{M}-\mathrm{H}$ & 1.44 & $\downarrow$ \\
\hline 19 & Phenylpyruvate & С9H8O3 & 4.47 & 163.0392 & $\mathrm{M}-\mathrm{H}$ & 1.61 & $\uparrow$ \\
\hline 20 & Riboflavin & C17H20N4O6 & 4.65 & 375.1285 & $\mathrm{M}-\mathrm{H}$ & 1.35 & $\downarrow$ \\
\hline 21 & Citric acid & C6H8O7 & 1.04 & 191.0194 & M-H & 1.88 & $\uparrow$ \\
\hline 22 & Leucine & C6H13NO2 & 4.20 & 130.0866 & $\mathrm{M}-\mathrm{H}$ & 2.58 & $\uparrow$ \\
\hline 23 & Creatine & C4H9N3O2 & 0.52 & 114.0669 & $\mathrm{M}-\mathrm{H}$ & 2.00 & $\uparrow$ \\
\hline 24 & Uric acid & $\mathrm{C} 5 \mathrm{H} 4 \mathrm{~N} 4 \mathrm{O} 3$ & 1.03 & 169.0203 & M-H & 1.29 & $\downarrow$ \\
\hline 25 & Linoleic acid & $\mathrm{C} 18 \mathrm{H} 32 \mathrm{O} 2$ & 4.23 & 280.2277 & M-H & 1.39 & $\uparrow$ \\
\hline 26 & Taurine & C2H7NO3S & 0.42 & 125.0152 & M-H & 1.52 & \\
\hline
\end{tabular}


Note: compared with the model group, ' $\uparrow$ ' refers to an upward trend and ' $\downarrow$ ' refers to a downward trend in the RSLD group.

\section{Figures}

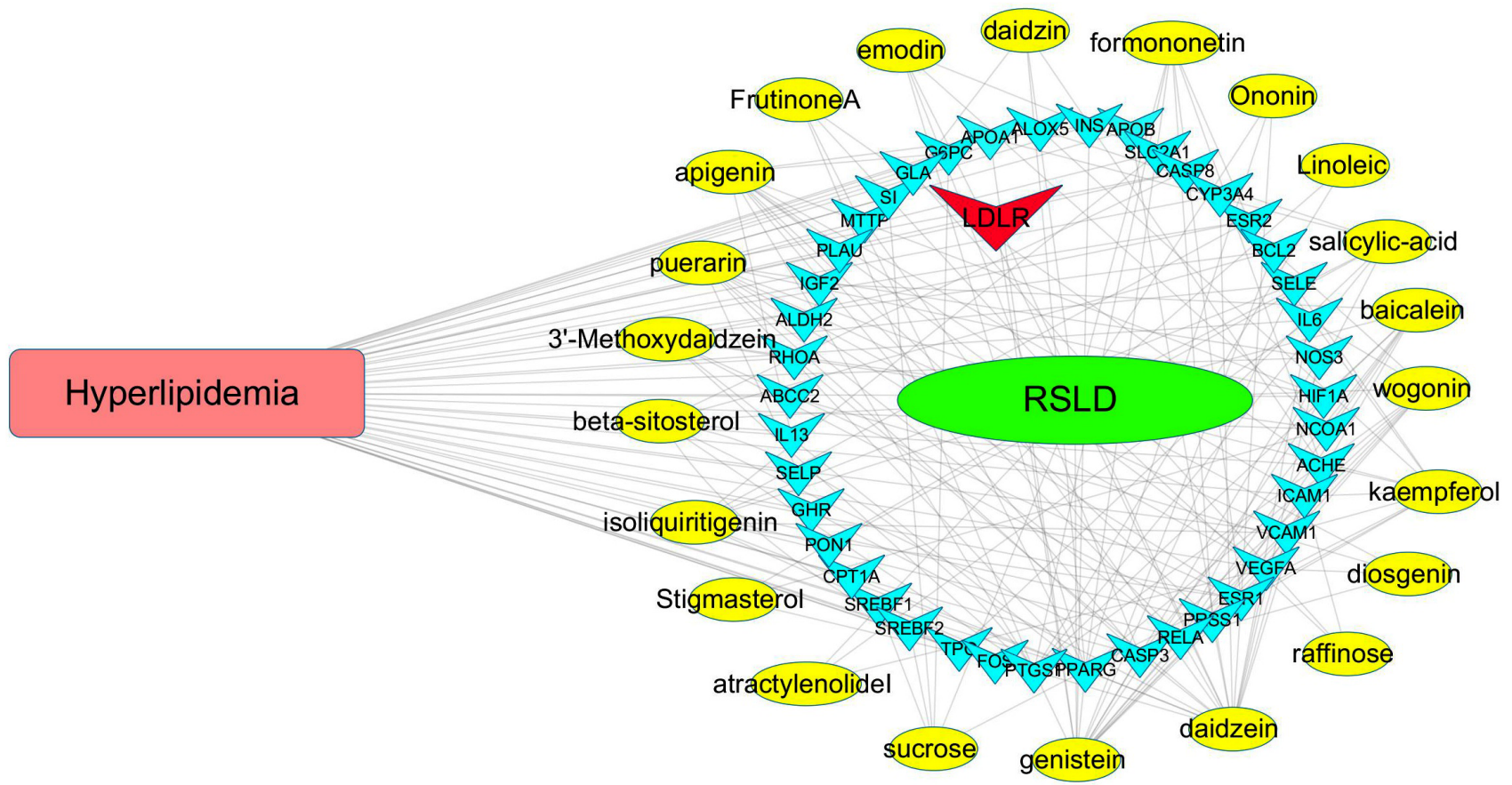

\section{Figure 1}

The component-target-disease network formed after RSLD intervention 


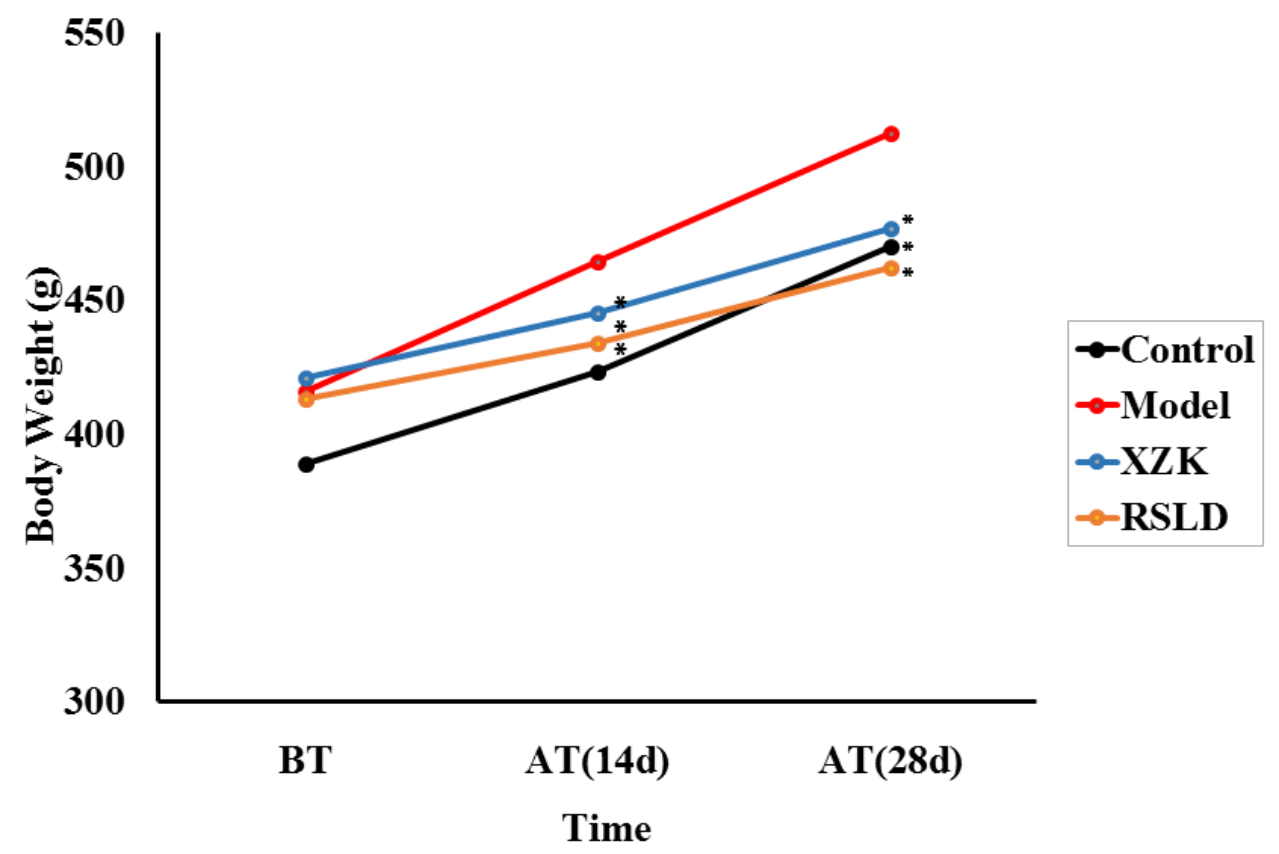

\section{Figure 2}

Body weight in each group $\llbracket \mathrm{x} \pm \mathrm{s} \rrbracket$ Note: Compared with the model group at the same time period, * $\mathrm{P}<$ 0.01 .

2

Figure 3

Blood lipid levels after treatment $₫ x \pm s \rrbracket$ Note: Compared with the model group at the same time period, * $P$ $<0.01$. 

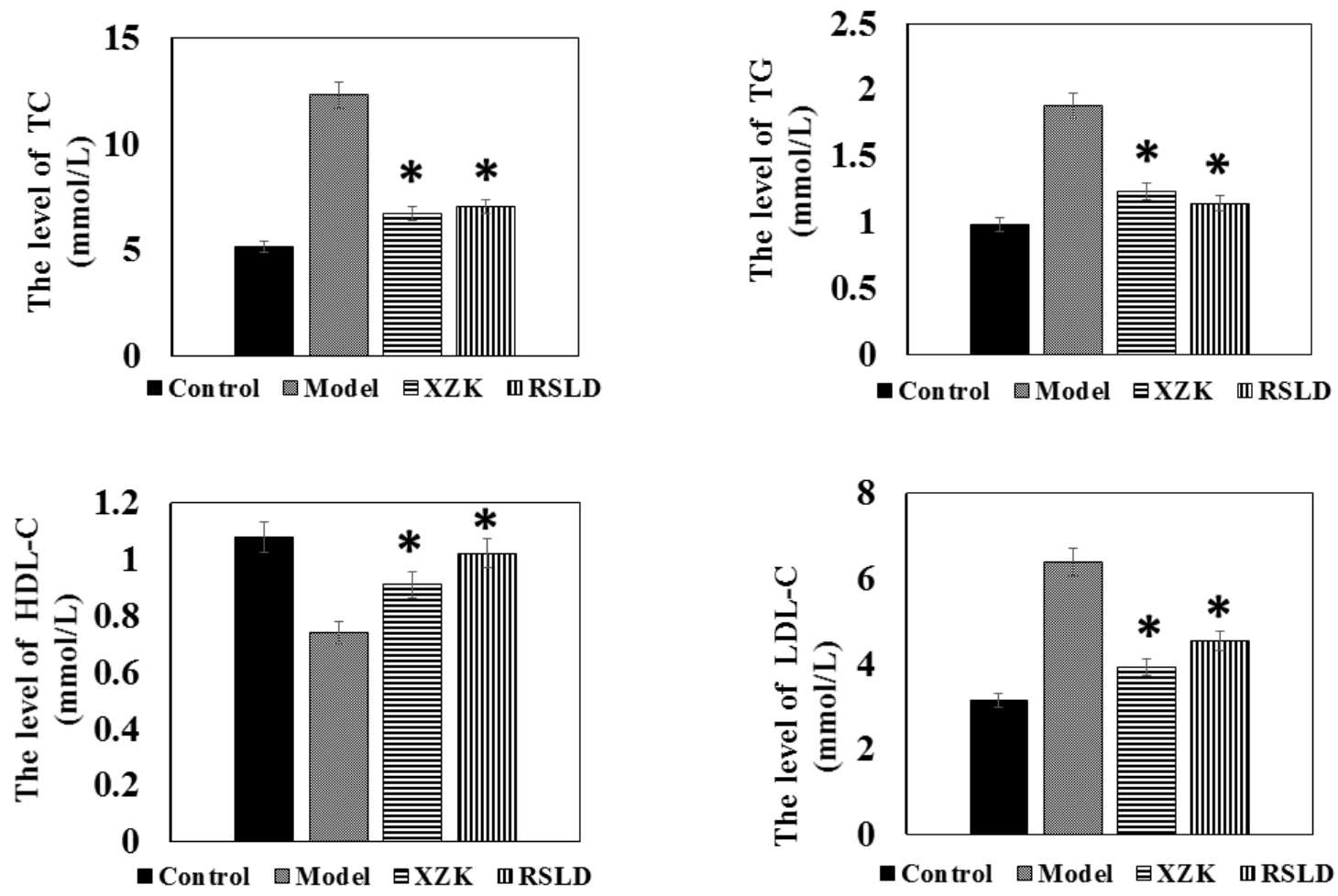

Figure 4

LDLR protein expression after treatment



Figure 5

Base peak intensity chromatogram

2

Figure 6

The OPLS-DA score plot after treatment

展

Figure 7

The cluster heat plot after treatment Note: $\mathrm{C}$ refers to control group, $\mathrm{M}$ refers to model group, $\mathrm{R}$ refers to RSLD group, $\mathrm{X}$ refers to XZK group. 


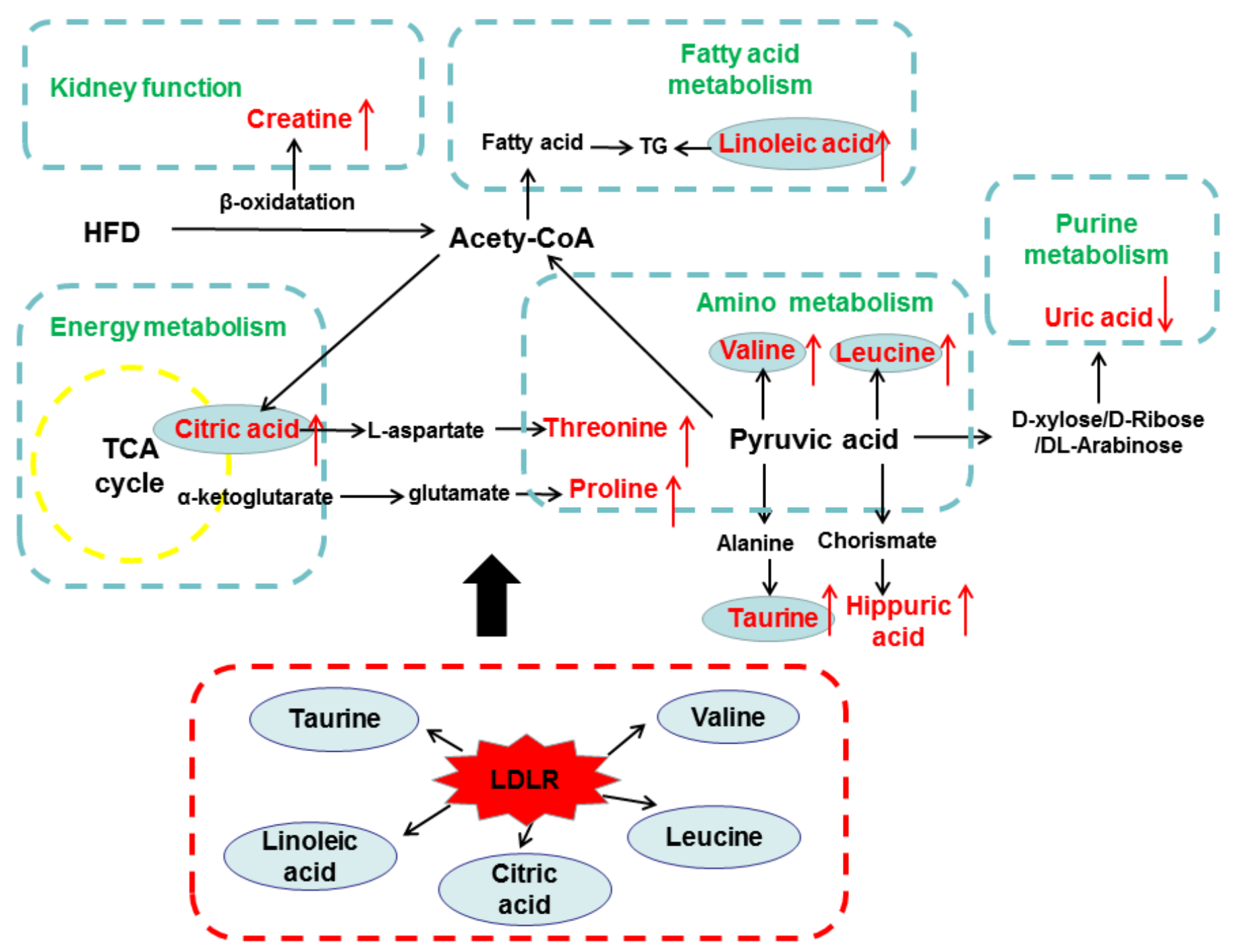

Figure 8

Potential metabolic pathways changed in hyperglycemic rats after treatment 


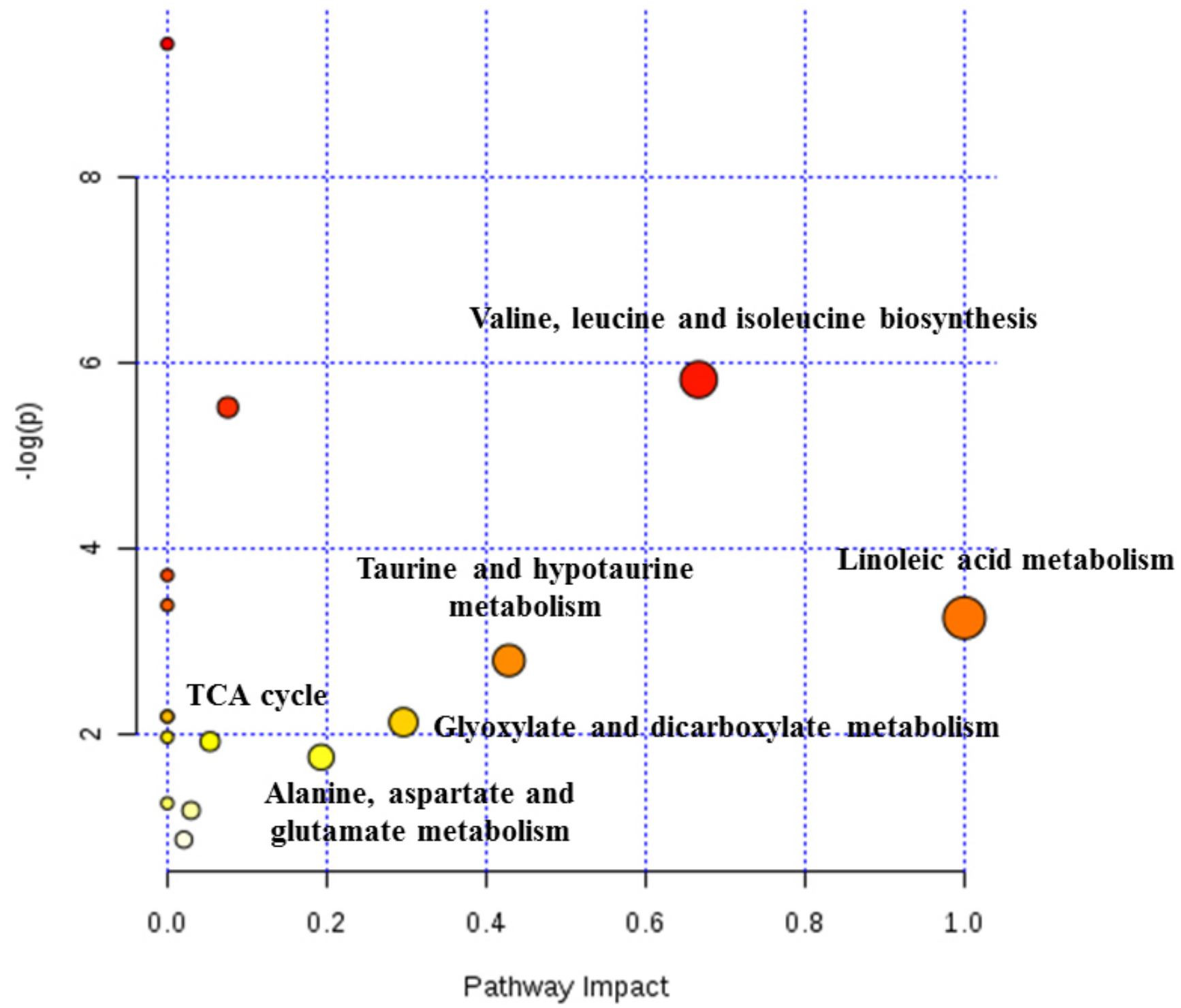

Figure 9

The pathways impacts regulated by RSLD 


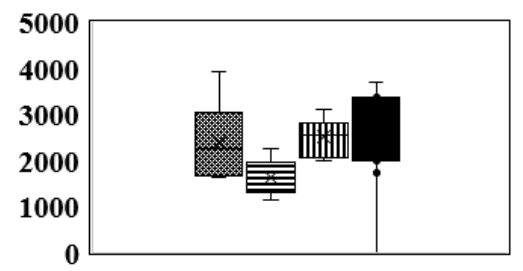

Citric acid

줐 Control $\boxminus$ Model

m XZK

- RSLD

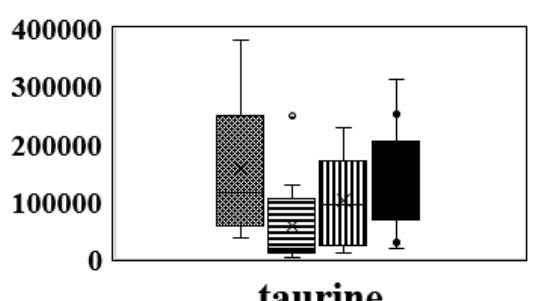

圈 Control $\boxminus$ Model

III XZK IRSLD

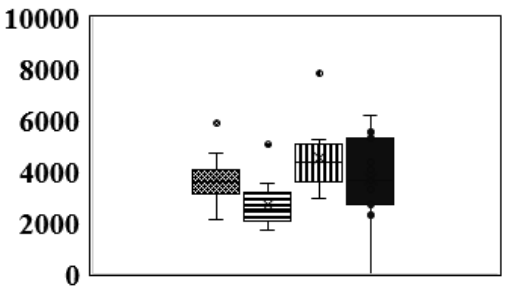

leucine

圈 Control घ Model

IIXZK RSLD

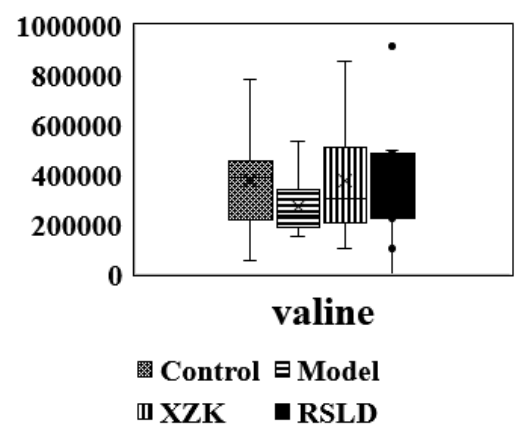

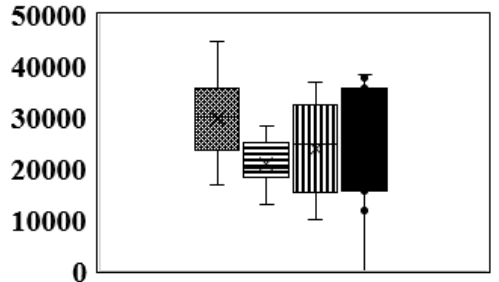

linoleic acid

图 Control $\boxminus$ Model

m XZK $\square$ RSLD

\section{Figure 10}

Comparison of the relative intensity of potential biomarkers regulated by LDLR Note: The blue frame represents the metabolic pathway, and the red frame represents potential biomarkers. Compared with the model group, ' $\uparrow$ 'refers to an upward trend and ' $\downarrow$ 'refers to an downward trend in RSLD group.

\section{Supplementary Files}

This is a list of supplementary files associated with this preprint. Click to download.

- TableS1.doc 